\title{
Características físico-químicas e composição centesimal de cortes cárneos de borregas confinadas e alimentadas com diferentes proporções volumoso: concentrado
}

\author{
Physicochemical characteristics and centesimal composition of ewe lambs meat cuts \\ with different ratio forage: concentrate
}

\author{
COUTINHO, Marcelo Aranda da Silva ${ }^{1 *}$; MORAIS, Maria da Graça ${ }^{1}$; ALVES, \\ Fabiana Villa ${ }^{2}$; FERNANDES, Henrique Jorge ${ }^{3}$; FEIJÓ, Gelson Luís Dias ${ }^{2}$; ÍTAVO, \\ Camila Celeste Brandão Ferreira ${ }^{1}$; COMPARIN, Marco Aurélio Scarton ${ }^{1}$; COELHO, \\ Roberta Gomes ${ }^{1}$
}

\footnotetext{
${ }^{1}$ Universidade Federal de Mato Grosso do Sul, Faculdade de Medicina Veterinária e Zootecnia, Campo Grande, Mato Grosso do Sul, Brasil.

${ }^{2}$ Embrapa Gado de Corte, Campo Grande, Mato Grosso do Sul, Brasil.

${ }^{3}$ Universidade Estadual de Mato Grosso do Sul, Aquidauana, Mato Grosso do Sul, Brasil.

*Endereço para correspondência: marceloscoutinho@hotmail.com
}

\section{RESUMO}

Objetivou-se avaliar as características físicoquímicas e a composição centesimal da carne de borregas confinadas recebendo diferentes relações volumoso (V): concentrado (C) (80:20; 60:40; 40:60 e 20:80) e os cortes cárneos destes animais (paleta, lombo e do pernil). Foram utilizadas 24 borregas (mestiças) recémdesmamadas com peso vivo inicial médio de $23,1 \pm 2,1 \mathrm{~kg}$, confinadas por 120 dias. Após o abate, foram removidos os músculos Triceps brachii (paleta), Longissimus dorsi (lombo) e Semimembranosus (pernil) para representar os respectivos cortes. $\mathrm{O}$ aumento nos níveis de $\mathrm{C}$ não influenciou a composição centesimal dos cortes cárneos e diminuiu a luminosidade $\left(\mathrm{L}^{*}\right)$ e aumentou a tonalidade vermelha $\left(\mathrm{a}^{*}\right)$. O lombo apresentou o menor teor de umidade (U) $(699,7 \mathrm{~g} / \mathrm{kg})$ e maior teor de extrato etéreo $(64,5 \mathrm{~g} / \mathrm{kg})$, enquanto a paleta apresentou maior teor de U $(738,7 \mathrm{~g} / \mathrm{kg})$ e menor teor de PB $(214,0 \mathrm{~g} / \mathrm{kg})$, e o pernil apresentou o maior valor para PB $(232,8 \mathrm{~g} / \mathrm{kg})$ e valores intermediários para U $(716,3 \mathrm{~g} / \mathrm{kg})$ e EE $(40,1 \mathrm{~g} / \mathrm{kg})$. O pernil forneceu a carne menos macia $(\mathrm{FC}=2,98 \mathrm{kgf})$. Para as características físico-químicas, o lombo e a paleta apresentaram carnes com maior valor de $b^{*}$ e o pernil e a paleta obtiveram maiores valores para $\mathrm{a}^{*}$. As diferentes proporções $\mathrm{V}: \mathrm{C}$ não alteram a composição centesimal $\mathrm{e}$ proporcionam valores diferentes para $\mathrm{L}^{*} \mathrm{e} \mathrm{a}^{*}$.
Os cortes cárneos apresentam diferenças na composição química e nas características físicas.

Palavras-chave: cor, força cisalhamento, lombo, paleta, pernil

\section{SUMMARY}

It was aimed to evaluate the physicochemical characteristics and centesimal composition of ewe lambs meat fed different forage (F):concentrate (C) ratio (80F:20C, 60F:40C, 40F:60C and 20F:80C) and meat cuts these animals (shoulder, loin and leg). Twenty-four of ewe lambs (crossbred) recently weaned with initial weight of $23.1 \pm 2.1 \mathrm{~kg}$ was feedlot period was 120 days. After the slaughter, Triceps brachii (shoulder), Longissimus dorsi (loin) and Semimembranosus (leg) muscles were removing to represent the respective meat cuts. Increased levels of $\mathrm{C}$ didn't influence the chemical composition of meat cuts and decreased the lightness $(\mathrm{L} *)$ and increased red color $(\mathrm{a} *)$. The loin had the lowest moisture content (M) $(699.7 \mathrm{~g} / \mathrm{kg})$ and higher content of ether extract (EE) $(64.5 \mathrm{~g} / \mathrm{kg})$, while the shoulder showed a higher content of $\mathrm{M}(738.7 \mathrm{~g} / \mathrm{kg})$ and lower content of CP $(214.0 \mathrm{~g} / \mathrm{kg})$. The leg had the highest value for $\mathrm{CP}(232.8 \mathrm{~g} / \mathrm{kg})$ and 
intermediate values for $\mathrm{M}(716.3 \mathrm{~g} / \mathrm{kg})$ and EE $(40.1 \mathrm{~g} / \mathrm{kg})$. For the physicochemical characteristics, the loin and the shoulder had meat with more $b^{*}$ while leg and the shoulder had more $\mathrm{a}^{*}$. The leg meat was less tender $(\mathrm{SF}=$ $2.98 \mathrm{kgf}$ ). The different ratios $\mathrm{V}: \mathrm{C}$ doesn't alter the composition and provide different values for $\mathrm{L}^{*}$ and $\mathrm{a}^{*}$. The meat cuts differ in chemical composition and physical characteristics.

Keywords: color, leg, loin, shear force, shoulder

\section{INTRODUÇÃO}

O confinamento para ruminantes deve ser considerado de forma estratégica para melhoria nos índices produtivos. A utilização desta ferramenta, além de outras vantagens, permite o abate de animais mais jovens, incrementando a produção de carnes de melhor qualidade físico-química e sensorial (PRIOLO et al., 2002). Entretanto, o custo deste sistema é elevado, principalmente devido a dietas mais onerosas. Com o intuito de se oferecer dietas com menor custo, a relação volumoso:concentrado (V:C) pode ser alterada com base nos preços dos ingredientes e no desempenho desejável aos animais.

Alguns trabalhos reportaram o efeito da relação V:C sobre o perfil lipídico (DEMIREL et al., 2006), rendimento de cortes cárneos e composição centesimal (CARVALHO \& MEDEIROS, 2010), bem como sobre alguns atributos sensoriais da carne de ovinos (COSTA et al., 2011b). Contudo, pouco se sabe da influência destas diferentes relações sobre a qualidade químico-física e a composição centesimal dos cortes cárneos de ovinos, em especial de borregas.

A paleta, o lombo e o pernil de ovinos são os cortes cárneos mais demandados pelos consumidores e representam, juntos, em média $66 \%$ da carcaça em cordeiros (PINHEIRO et al., 2009a). Os cortes podem diferir e apresentar comportamentos distintos em seus atributos. Assim, a caracterização destes cortes auxiliaria os consumidores quanto as suas preferências e também nas tomadas de decisões das indústrias de carne.

Com a melhoria no sistema de produção, a presença de borregas e fêmeas de descarte na terminação tem aumentado consideravelmente. Com isso, estas categorias têm adquirido relevância em estudos que avaliam diferentes dietas sobre o desempenho animal e características de carcaça (COSTA et al., 2012; DAVID et al., 2012; SANTOS et al., 2011). Porém, estudos que objetivam avaliar qualidade dos cortes de fêmeas recebendo diferentes relações volumoso:concentrado são escassos e necessários.

Deste modo, objetivou-se avaliar as características físico-químicas e a composição centesimal das carnes da paleta, lombo e pernil de borregas confinadas recebendo diferentes níveis de concentrado.

\section{MATERIAIS E MÉTODOS}

Foram utilizadas 24 borregas recémdesmamadas, com aproximadamente três meses de idade, mestiças SRD, oriundas de cruzamento com animais lanados, com peso vivo inicial médio de $23,1 \pm 2,1 \mathrm{~kg}$. Os animais foram alojados em baias individuais com $3,0 \mathrm{~m}^{2}$. Cada seis animais receberam, aleatoriamente, uma dieta com um dos quatro níveis de concentrado: dietas com 20, 40, 60 e $80 \%$ de concentrado em base da matéria seca. As dietas consistiram de volumoso à base de feno de Tifton 85 (Cynodon spp.) moído, e concentrado comercial composto por milho, farelo de soja, levedura seca de cervejaria e melaço de 
cana em pó. As dietas foram formuladas conforme o NRC (2007) de acordo com os níveis crescentes de concentrado (Tabela 1). Todos os animais receberam alimentação ad libitum, fornecida duas vezes ao dia (8 e às 16 horas).

A recria das borregas compreendeu do peso corporal médio de $23,1 \pm 2,1 \mathrm{~kg}$ até ao peso corporal em jejum de $28,5 \pm$ 1,$9 ; 31,7 \pm 3,4 ; 34,7 \pm 1,8$ e $37,4 \pm$ 2,6kg para os níveis de 20, 40, 60 e 80\% concentrado, respectivamente. A partir destes pesos, iniciou-se a terminação que se prolongou até que o tempo total de confinamento (recria + terminação) fosse de 120 dias, com as borregas atingindo o peso corporal ao abate de $39,4 \pm 2,9 ; 42,3 \pm 5,1 ; 46,0 \pm 2,7$ e 50,3 $\pm 3,9 \mathrm{~kg}$ para os níveis de $20,40,60$ e $80 \%$ concentrado, respectivamente.

Tabela 1. Composição químico-bromatológica e nutrientes digestíveis totais dos alimentos e das dietas experimentais expressos na matéria seca

\begin{tabular}{lclllll}
\hline \multirow{2}{*}{ Componentes (\% da MS) } & \multicolumn{2}{c}{ Alimentos } & \multicolumn{4}{c}{$\begin{array}{c}\text { Dieta total } \\
\text { Níveis }\end{array}$} \\
& \multicolumn{1}{c}{ de concentrado nas dietas } \\
\cline { 2 - 7 } & Feno & Concentrado & 20 & 40 & 60 & 80 \\
\hline Matéria seca & 92,0 & 90,6 & 91,7 & 91,5 & 91,2 & 90,9 \\
Matéria orgânica & 94,0 & 93,9 & 94,0 & 93,9 & 93,9 & 93,9 \\
Proteína bruta & 9,92 & 2,53 & 13,7 & 16,5 & 19,3 & 22,2 \\
Extrato etéreo & 2,05 & 3,11 & 2,26 & 2,47 & 2,69 & 2,90 \\
Fibra em detergente neutro & 78,7 & 28,7 & 68,7 & 58,7 & 48,7 & 38,7 \\
Fibra em detergente ácido & 43,8 & 5,92 & 36,2 & 28,7 & 21,1 & 13,5 \\
Lignina & 5,46 & 0,41 & 4,45 & 3,44 & 2,43 & 1,42 \\
Carboidratos não fibrosos & 3,36 & 36,8 & 9,34 & 16,2 & 23,2 & 30,1 \\
NDT $^{1}$ & - & - & 64,0 & 72,0 & 77,0 & 77,0 \\
NDT $^{2}$ & - & - & 68,0 & 73,0 & 77,0 & 76,0 \\
\hline
\end{tabular}

Fonte: adaptado de Ribeiro (2011).

NDT = nutrientes digestíveis totais obtidos por ensaio de digestibilidade na recria (1) e na terminação (2).

No final do período de alimentação, as borregas foram submetidas a 16 horas de jejum de sólidos e em seguida abatidas. As carcaças foram seccionadas ao meio e armazenadas em câmara frigorífica à temperatura inferior a $5^{\circ} \mathrm{C}$ por 24 horas. Em seguida, os cortes cárneos da meia carcaça direita foram acondicionados em sacos plásticos e armazenados em freezer a $-18^{\circ} \mathrm{C}$ para posteriores análises. Os músculos representativos da paleta (Triceps brachii), lombo (Longissimus dorsi) e pernil (Semimembranosus) foram seccionados, sendo retiradas duas amostras de $2,5 \mathrm{~cm}$ de espessura (em forma de bife e sem gordura de cobertura) da parte medial dos mesmos para determinação do $\mathrm{pH}$, cor, perda por cocção (PPC) e força de cisalhamento (FC). As amostras, logo que obtidas, tiveram o $\mathrm{pH}$ e a temperatura aferidos por meio de potenciômetro portátil HI99163 (Hanna instruments brasil Inc, São Paulo, Brasil).

Após a exposição da superfície das amostras ao ambiente por cerca de 30 minutos, realizaram-se as avaliações da cor por meio de espectrofotômetro Hunter Lab Mini Scan XE Plus 45/0-L 
(Reston, VA, EUA), utilizando o iluminante A, 10 graus para observação padrão, calibrado para dois padrões (preto e branco). Utilizou-se a escala CIELAB (onde, $\mathrm{L}^{*}$ corresponde à luminosidade; $\mathrm{a}^{*}$ à tonalidade de vermelho e $b^{*}$ à de amarelo) para as determinações.

Para avaliação das PPC, as amostras foram pesadas antes (PI) e após (PF) serem submetidas ao cozimento em forno elétrico pré-aquecido, sob temperatura de $300^{\circ} \mathrm{C}$, durante 5 minutos para cada lado da amostra. As amostras apresentavam temperatura de $71^{\circ} \mathrm{C}$ na porção mediana logo que retiradas do forno. A PPC foi obtida por meio da seguinte fórmula: PPC $(\%)=$ $[(\mathrm{PI}-\mathrm{PF}) / \mathrm{PI}] \times 100$, onde $\mathrm{PPC}$ é perda por cocção; PI é peso inicial; e PF é peso final.

Das amostras assadas foram retiradas subamostras cilíndricas para determinação da FC (WHEELER et al., 1997). O cisalhamento foi realizado em um texturômetro da marca TA XT PLUS (Stable Micro Systems, Godalming, Reino Unido), perpendicularmente na orientação longitudinal das fibras musculares. Cada amostra foi cisalhada por completo em seu centro geométrico por um dispositivo "Warner-Bratzler 'V' slot blade" (espessura de 3,0mm e abertura triangular de $60^{\circ}$ ). Foi utilizada uma célula com carga de $30 \mathrm{~kg}$ de compressão e velocidade de $20 \mathrm{~cm} / \mathrm{min}$, sendo o valor de FC medido em Kgf.

A fração remanescente do músculo foi moída, homogeneizada e armazenada para a avaliação dos teores de umidade, matéria mineral $(\mathrm{MM})$, proteína bruta (PB) e extrato etéreo (EE), de acordo com AOAC (1995).

Os dados foram analisados em um delineamento inteiramente casualizado, com arranjo fatorial $3 \times 4$ (três cortes cárneos x quatro níveis de concentrado). $\mathrm{O}$ modelo inicial incluiu, assim, os efeitos de corte cárneo, e os efeitos linear e quadrático do nível de concentrado, além das interações. Todas as interações foram avaliadas e retiradas do modelo por serem não significativas. Assim, o modelo final de análise avaliou separadamente o efeito dos cortes cárneos, e os efeitos lineares e quadráticos do nível de concentrado. Quando identificado efeito significativo de corte cárneo, utilizou-se o teste de Tukey na comparação das médias dos diferentes cortes. Utilizou-se o PROC GLM do SAS v 9.3 (STATISTICAL ANALYSIS SYSTEM, 2011) em todas as análises estatísticas. Adotou-se nível de significância de $5 \%$.

\section{RESULTADOS E DISCUSSÃO}

$\mathrm{Na}$ recria, o consumo de matéria seca (g/dia) e o ganho médio diário (g/dia) foram $1196 \pm 28$ e $135 \pm 14 ; 1139 \pm 23$ e $168 \pm 11 ; 1177 \pm 23$ e $218 \pm 11 ; 1193$ \pm 28 e $246 \pm 13$ para os níveis de 20,40 , 60 e $80 \%$ concentrado, respectivamente. Enquanto que na terminação foram $1450 \pm 41$ e $162 \pm 19 ; 1328 \pm 31$ e $159 \pm 15 ; 1295 \pm 31$ e $162 \pm 15 ; 1207 \pm$ 41 e $171 \pm 19$. Maiores detalhes de desempenho estão disponíveis no trabalho de Ribeiro (2011).

Não foi observada interação $(\mathrm{P}>0,05)$ entre cortes cárneos e os níveis de concentrado para as variáveis estudadas. Os cortes estudados apresentaram comportamento semelhante com aumento da participação de concentrado na dieta. Isso demonstra que a alteração da relação $\mathrm{V}$ : $\mathrm{C}$ não afeta apenas a qualidade de determinados cortes.

Não se observou efeito linear ou quadrático $(\mathrm{P}>0,05)$ do nível de concentrado sobre a composição centesimal das carnes (Tabela 2). Os valores médios para umidade, $\mathrm{MM}, \mathrm{PB}$ 
e EE foram de 71,$83 ; 1,22 ; 22,4$; e $4,82 \mathrm{~g} / 100 \mathrm{~g}$ de carne, respectivamente. De modo geral, as carnes das borregas aqui avaliadas apresentaram menor teor de umidade, maior de PB e de EE, e teores de MM similares aos observados nos trabalhos com que apresentaram manejo nutricional semelhante (CARVALHO \& MEDEIROS, 2010;
LEÃO et al., 2011; ZEOLA et al., 2004). O maior teor de EE nas carnes de borregas pode ser atribuído a diferenças de raças, sexo, dieta e idade existente entre os trabalhos. Normalmente, as demais variáveis (umidade e PB) estão inversamente relacionadas com o teor de EE na carne (BONAGURIO et al., 2004; PFLANZER \& FELÍCIO, 2011).

Tabela 2. Composição centesimal das carnes de borregas confinadas recebendo diferentes níveis de concentrado na dieta

\begin{tabular}{|c|c|c|c|c|c|c|c|}
\hline \multirow{2}{*}{$\begin{array}{l}\text { Variável } \\
\text { (g/100g carne) }\end{array}$} & \multicolumn{4}{|c|}{ Nível de Concentrado na Dieta (\%) } & \multirow{2}{*}{$\begin{array}{l}\text { CV } \\
(\%)\end{array}$} & \multicolumn{2}{|c|}{ Valor - p Efeito } \\
\hline & 20 & 40 & 60 & 80 & & Linear & Quadrático \\
\hline Umidade & 72,5 & 71,3 & 71,7 & 71,7 & 1,96 & 0,075 & 0,112 \\
\hline Matéria Mineral & 1,26 & 1,25 & 1,20 & 1,16 & 20,50 & 0,999 & 0,805 \\
\hline Proteína Bruta & 22,1 & 22,2 & 22,5 & 22,6 & 2,50 & 0,619 & 0,957 \\
\hline Extrato Etéreo & 4,48 & 5,42 & 4,82 & 4,55 & 31,73 & 0,115 & 0,096 \\
\hline
\end{tabular}

$\mathrm{CV}=$ coeficiente de variação.

Apesar do longo período de confinamento com dietas que proporcionaram diferentes ganhos médio diário e pesos de abate, não foram observadas diferenças na composição centesimal. Isto não era esperado, pois, as diferentes taxas de ganho geralmente conferem composição química dos ganhos distinta, o que afetaria a composição centesimal das carnes. Outros estudos, no entanto, que também avaliaram diferentes níveis de concentrado na dieta dos animais também não encontraram diferenças expressivas na composição centesimal das carnes (LEÃO et al., 2011; ZEOLA et al., 2004). Resultados semelhantes foram ainda obtidos por Carvalho \& Medeiros (2010) que avaliaram quatro níveis de energia na dieta $(1,49 ; 1,55$; 1,62 ; e $1,68 \mathrm{Mcal}$ de energia líquida $/ \mathrm{kg}$ de MS) e não observaram diferenças na composição centesimal da carne de cordeiros.

Os trabalhos supracitados abateram os animais de tratamentos distintos com pesos de abate semelhantes, em contraste com o atual estudo, onde os animais com os maiores níveis de concentrado na dieta tinham os maiores pesos corporais ao abate. Segundo Bonagurio et al. (2004), com aumento do peso do animal há redução percentual na umidade, na PB e na MM, e que isso ocorre em função do aumento do tecido adiposo quando se aproxima da maturidade. Neste sentido, pode-se sugerir que as borregas com os maiores níveis de concentrado (maiores pesos ao abate) não alcançaram a maturidade ou não possuíam potencial genético para incrementar a deposição de gordura intramuscular, e deste modo, não interferindo nos teores dos demais componentes.

Na comparação entre os cortes cárneos quanto à composição centesimal (Tabela 3), de maneira geral, os resultados aqui obtidos atenderam o pressuposto de que o teor de lipídios apresenta uma correlação negativa com a umidade e a PB (BONAGURIO et al., 
2004; PFLANZER \& FELÍCIO, 2011). A paleta apresentou maior umidade, seguida pelo pernil e pelo lombo. Para a $\mathrm{PB}$, o pernil apresentou o maior valor, e a paleta o menor. $O$ teor de EE no lombo foi superior $(\mathrm{P}<0,05)$ ao da paleta e ao do pernil, os quais não diferiram $(\mathrm{P}>0,05)$. Observa-se que dentre as variáveis avaliadas, o extrato etéreo apresentou a diferença mais expressiva entre os cortes cárneos. $\mathrm{O}$ lombo exibiu teores de EE em torno de $60 \%$ superior ao da paleta e do pernil. Quanto à MM, observou-se que o lombo apresentou o menor teor $(\mathrm{P}<0,05)$.

Tabela 3. Composição centesimal dos cortes cárneos de borregas confinadas recebendo diferentes níveis de concentrado na dieta

\begin{tabular}{lcccc}
\hline \multirow{2}{*}{ Variável (g/100 g carne) } & \multicolumn{3}{c}{ Cortes $^{1}$} & \multirow{2}{*}{ CV $(\%)$} \\
\cline { 2 - 4 } & Lombo & Paleta & Pernil & \\
\hline Umidade & $69,9^{\mathrm{c}}$ & $73,8^{\mathrm{a}}$ & $71,6^{\mathrm{b}}$ & 1,96 \\
Matéria Mineral & $1,04^{\mathrm{b}}$ & $1,23^{\mathrm{a}}$ & $1,39^{\mathrm{a}}$ & 20,50 \\
Proteína Bruta & $22,4^{\mathrm{b}}$ & $21,4^{\mathrm{c}}$ & $23,2^{\mathrm{a}}$ & 2,50 \\
Extrato Etéreo & $6,45^{\mathrm{a}}$ & $3,98^{\mathrm{b}}$ & $4,01^{\mathrm{b}}$ & 31,73 \\
\hline
\end{tabular}

${ }^{1}$ Médias seguidas por letras diferentes nas linhas diferem $(\mathrm{P}<0,05)$ pelo teste Tukey.

$\mathrm{CV}=$ coeficiente de variação.

Os diferentes níveis de concentrado na dieta não afetaram $(\mathrm{P}>0,05)$ as $\mathrm{PPC}$ (Tabela 4). A composição química média do lombo de cordeiros terminados com diferentes dietas foi 72,$75 ; 1,11 ; 20,34 \mathrm{e}$ $6,54 \mathrm{~g} / 100 \mathrm{~g}$ para umidade, MM, PB e EE respectivamente, no trabalho de Madruga et al. (2005). Pinheiro et al. (2008) observaram na paleta de cordeiros valores de 74,05; 5,36; 18,85 e 1,15\% para umidade, EE, PB e MM, respectivamente. Costa et al. (2011a) avaliaram ovinos machos não castrados e observaram os seguintes valores médios para o pernil: $75,24 \%$ de umidade; $1,06 \%$ de MM; $18,49 \%$ de PB e $3,75 \%$ de EE. Com base nestes dados e os aqui obtidos, o lombo parece ter o maior teor de EE entre os principais cortes cárneos em ovinos, independentemente do nível de concentrado na dieta (interação $\mathrm{P}>0,05$ ). Isso sugere que o lombo possui maior precocidade ou/e é predisposto geneticamente para deposição de gordura intramuscular, logo, é associado com menores teores de umidade e PB.

Este resultado pode ter sido influenciado pela ausência de diferença no teor de EE nas carnes. Segundo Miller (2004), a gordura presente na carne pode atuar como um isolante contra a perda de umidade. Corroborando com esta hipótese, Sañudo et al. (1997) apresentaram resultados onde carnes com menores PPC exibiam os maiores teores de gordura. Por outro lado, o teor de umidade semelhante entre as carnes também pode explicar esta falta de diferença nas PPC, uma vez que o maior teor de umidade nas carnes mais magras predispõe a maiores PPC. Pinheiro et al. (2009a), em estudos com cordeiros, também não verificaram diferença na PPC quando o teor de EE e de umidade permaneceram constantes na carne, mesmo com aumento dos níveis de concentrado.

Apesar da metodologia adotada no presente experimento ter utilizado temperatura de $300^{\circ} \mathrm{C}$ para cocção, em 
contraste com temperaturas em torno de $170-200^{\circ} \mathrm{C}$ descrita em alguns trabalhos da literatura (COSTA et al., 2011b; MORENO et al., 2011; PINHEIRO et al., 2009a, 2009b), os valores de PPC aqui obtidos estão de acordo com os observados naqueles estudos. Talvez, o fato das amostras apresentarem temperaturas em torno de $71^{\circ} \mathrm{C}$ ao final da cocção, semelhante à relatada nos demais trabalhos, possibilitaram resultados similares.

Tabela 4. Características físico-químicas das carnes de borregas confinadas recebendo diferentes níveis de concentrado na dieta

\begin{tabular}{|c|c|c|c|c|c|c|c|}
\hline \multirow{2}{*}{ Variável } & \multicolumn{4}{|c|}{ Nível de Concentrado na Dieta (\%) } & \multirow{2}{*}{$\begin{array}{l}\mathrm{CV} \\
(\%)\end{array}$} & \multicolumn{2}{|c|}{ Valor - P Efeito } \\
\hline & 20 & 40 & 60 & 80 & & Linear & Quadrático \\
\hline $\operatorname{PPC~}(\%)$ & 42,68 & 40,27 & 38,13 & 37,00 & 8,65 & 0,096 & 0,433 \\
\hline $\mathrm{pH}$ & 5,87 & 5,86 & 5,85 & 5,93 & 2,41 & 0,322 & 0,220 \\
\hline $\mathrm{TpH}\left({ }^{\circ} \mathrm{C}\right)$ & 21,21 & 22,38 & 22,02 & 21,92 & 11,86 & 0,263 & 0,299 \\
\hline $\mathrm{L}^{*}$ & 44,91 & 42,64 & 42,68 & 42,56 & 6,39 & 0,047 & 0,103 \\
\hline$a^{*}$ & 22,72 & 23,50 & 23,38 & 23,36 & 4,15 & 0,046 & 0,080 \\
\hline$b^{*}$ & 15,63 & 15,90 & 15,96 & 16,30 & 8,14 & 0,889 & 0,902 \\
\hline FC (kgf) & 3,00 & 2,39 & 2,22 & 1,93 & 26,48 & 0,061 & 0,294 \\
\hline
\end{tabular}

FC = força cisalhamento de carne assada, demais parâmetros: carne in natura, $\mathrm{PPC}=$ perda de peso por cocção, TpH = temperatura da amostra no momento da mensuração do $\mathrm{pH}, \mathrm{L}^{*}=$ luminosidade, $\mathrm{a}^{*}=$ intensidade de vermelho, $\mathrm{b}^{*}=$ intensidade de amarelo e $\mathrm{FC}=$ força de cisalhamento.

$\mathrm{CV}=$ coeficiente de variação.

$\mathrm{O} \mathrm{pH}$ e o $\mathrm{TpH}$ também não diferiram entre os níveis de concentrado. Resultado semelhante para o $\mathrm{pH}$ foi observado por Costa et al. (2011b), que avaliaram dois níveis de concentrado (20V:80C e 50V:50C) em três grupos genéticos. Segundo Gonçalves et al. (2004), a carne ovina raramente apresenta grandes variações no $\mathrm{pH}$. Uma explicação para isso seria a relação entre os diferentes tipos de fibras que compõe a carne ovina, que segundo Eikelenboom et al. (2004), é um importante fator na taxa e extensão do declínio do $\mathrm{pH}$ no estabelecimento do rigor mortis. Além disso, os diferentes níveis de concentrado provavelmente possibilitaram condições fisiológicas semelhantes ante mortem, não interferindo nas reservas e na quebra do glicogênio post mortem e com isso, conferindo $\mathrm{pH}$ semelhante entre os tratamentos.
No presente estudo, o aumento do concentrado na dieta provocou uma diminuição linear $(\mathrm{P}<0,05)$ nos valores de $\mathrm{L}^{*}$ e um aumento linear nos valores de $a^{*}$. Em contraste, Costa et al. (2011b) não encontraram diferença significativa para $L^{*}(23,1)$ e a* $(8,7)$ na avaliação de duas relações V:C (20:80 e 50:50), mas encontraram diferença significativa para b* $(20,9$ x 21,4$)$, com maior valor para o nível mais elevado de concentrado. Segundo estes autores, o valor elevado para $b^{*}$ pode ter sido decorrente do elevado teor de lipídios na carne. Esta suposição pode ser aplicada para o presente experimento que apresentou valores relativamente elevados $\mathrm{e}$ consistentes de EE e b* nos depósitos estudados. Assim, a falta de diferença nos valores de $b^{*}$ entre os níveis de concentrado pode ser creditada aos semelhantes teores de EE nos tratamentos. O comportamento dos 
resultados de $\mathrm{L}^{*}$ e a* com aumento de concentrado na dieta pode ser justificado pela diferença de peso entre os animais ao abate. Segundo Bonagurio et al. (2003), o maior desenvolvimento muscular nos animais mais pesados, aumenta a quantidade de mioglobina, interferindo no valor de a*. Além disso, há incremento nos depósitos de gordura, os quais reduzem o teor de água e, consequentemente, tem-se menor luminosidade (menor $\mathrm{L}^{*}$ ). $\mathrm{Na}$ avaliação da $\mathrm{FC}$, não foi verificada diferença significativa $(\mathrm{P}>0.05)$ entre as diferentes proporções V:C (Tabela 4). A FC é um método de avaliação objetiva da maciez da carne, que tem sido utilizada com maior frequência nas pesquisas em ciências de carnes. Bickerstaffe et al. (2001) classificaram a carne ovina como macia, quando esta apresenta FC menor que $8 \mathrm{kgf}$. Desta forma, a FC da carne cozida das borregas aqui estudadas, para todos os níveis de concentrado foram muito macias. Segundo Warner et al. (2010), esta variável apresenta herdabilidade moderada e é afetada por múltiplos fatores ambientais, tendo o nível de concentrado efeito pequeno ou nulo como foi evidenciado no presente experimento. As características físicoquímicas das carnes das borregas nas diferentes relações $\mathrm{V}: \mathrm{C}$, de maneira geral, foram semelhantes. Observaramse pequenas variações na cor, onde, com o incremento do concentrado, as carnes tornaram-se menos claras e com maior valor de $a^{*}$.

$\mathrm{Na}$ avaliação das características físicoquímicas entre os cortes cárneos, as PPC foram semelhantes (Tabela 5), com valor médio de $39,5 \%$. No trabalho de Vieira et al. (2010) esta medida variou de 21,6 a $25,3 \%$ entre os tratamentos estudados. Já no estudo de Pinheiro et al. (2009b) os valores variaram de $37,31 \%$ no músculo Semimembranosus de ovelhas a $67,58 \%$ no músculo Triceps de cordeiros. Os autores sugeriram que esta diferença poderia ser devido aos diferentes teores de gordura entre os cortes. Contudo, esta justificativa não pode ser adotada para os resultados aqui obtidos, pois o lombo apresentou o maior teor de EE e menor umidade, mas apresentou PPC semelhante aos demais cortes. Talvez, as diferenças para EE e umidade verificadas nos cortes cárneos não foram suficientes para interferir nos valores de PPC.

Tabela 5. Característica físico-química dos cortes cárneos de borregas confinadas recebendo diferentes níveis de concentrado na dieta

\begin{tabular}{lcccc}
\hline \multirow{2}{*}{ Variável } & \multicolumn{3}{c}{ Cortes } & C.V. \\
\cline { 2 - 4 } & Lombo & Paleta & Pernil & $(\%)$ \\
\hline PPC, $\%$ & 40,1 & 39,7 & 38,7 & 8,65 \\
$\mathrm{pH}$ & $5,76^{\mathrm{b}}$ & $6,01^{\mathrm{a}}$ & $5,85^{\mathrm{b}}$ & 2,41 \\
$\mathrm{TpH},{ }^{\circ} \mathrm{C}$ & $23,26^{\mathrm{a}}$ & $19,15^{\mathrm{b}}$ & $23,24^{\mathrm{a}}$ & 11,85 \\
$\mathrm{~L}^{*}$ & 43,94 & 42,87 & 42,78 & 6,39 \\
$\mathrm{a}^{*}$ & $22,81^{\mathrm{b}}$ & $23,52^{\mathrm{a}}$ & $23,39^{\mathrm{a}}$ & 4,15 \\
$\mathrm{~b}^{*}$ & $15,61^{\mathrm{b}}$ & $15,53^{\mathrm{b}}$ & $16,69^{\mathrm{a}}$ & 8,14 \\
FC, kgf & $2,15^{\mathrm{b}}$ & $2,02^{\mathrm{b}}$ & $2,98^{\mathrm{a}}$ & 26,48 \\
\hline
\end{tabular}

$\mathrm{FC}=$ força cisalhamento de carne assada, demais parâmetros: carne in natura, $\mathrm{PPC}=$ perda de peso por cocção, $\mathrm{TpH}=$ temperatura da amostra no momento da mensuração do $\mathrm{pH}, \mathrm{L}^{*}=$ luminosidade, $\mathrm{a}^{*}=$ intensidade de vermelho, $\mathrm{b}^{*}=$ intensidade de amarelo e $\mathrm{FC}=$ força de cisalhamento.

Médias seguidas por letras diferentes nas linhas diferem $(\mathrm{P}<0,05)$ pelo teste Tukey.

$\mathrm{CV}=$ coeficiente de variação. 
A paleta obteve o maior e menor valores entre os cortes cárneos $(\mathrm{P}<0,05)$ para $\mathrm{pH}$ e $\mathrm{TpH}$, respectivamente. Já o pernil e o lombo não diferiram $(\mathrm{P}>0,05)$ para estas variáveis. $\mathrm{O}$ maior valor de pH para a paleta pode ser relacionado à menor temperatura no momento da aferição, como verificado por Jansen (2001). Alterações no pH da carne, normalmente, afetam outras variáveis físico-químicas, o que não ocorreu no presente estudo.

$\mathrm{O}$ valor do $\mathrm{pH}$ final na carne ovina de boa qualidade normalmente varia de 5,5 a 5,8 . Valores altos $(\mathrm{pH} \geq 6,0)$ podem ocasionar carne DFD (Dark, Firm and Dry) (HONIKEL, 2004). No presente trabalho, esta variável apresentou-se entre 5,76 a 6,01 entre os diferentes cortes cárneos avaliados (Tabela 5). Este intervalo não afetou negativamente os cortes cárneos, pois se apresentaram de boa qualidade de acordo com os resultados de cor, PPC (uma avaliação da capacidade de retenção de água da carne) e pela observação do aspecto da carne (variável não apresentada).

$\mathrm{O}$ parâmetro $\mathrm{L}^{*}$ não diferiu $(\mathrm{p}>0,05)$ entre os cortes, apresentando o valor médio de 43,20. Já os parâmetros a* e $\mathrm{b}^{*}$ apresentaram diferença significativa $(\mathrm{P}<0,05)$ entre os cortes. O parâmetro $\mathrm{a}^{*}$ teve os maiores valores no pernil e na paleta, e o $b^{*}$ no pernil. A paleta e o lombo não diferiram para $b^{*}$. Pinheiro et al. (2009b) avaliaram os mesmos cortes do presente estudo em três categorias animal de ovinos, e da mesma forma, não verificaram variações em $\mathrm{L}^{*}$, mas apenas para $\mathrm{a}^{*} \mathrm{e}$ $b^{*}$. Desta forma, verifica-se que $a^{*}$ e $b^{*}$ apresentam diferenças entre os cortes cárneos mais comumente que $\mathrm{L}^{*}$.

$\mathrm{O}$ valor de $\mathrm{L}^{*}$ é normalmente relacionado ao $\mathrm{pH}$ e ao teor de umidade na carnes. Carnes com maior $\mathrm{pH}$ apresentam maior capacidade de retenção de água, conferindo maior umidade na carne. Esta condição atua como uma barreira para a difusão de oxigênio, além disso, o pH elevado torna as enzimas citocromo mais ativas em consumir parte do oxigênio remanescente no músculo. Segundo Lawrie (2005), tais condições aumentam a concentração de desoximioglobina (cor vermelho púrpura), conferindo aspecto de carne mais escura. Talvez, as diferenças entre os cortes para umidade e $\mathrm{pH}$ não foram suficientes para alterar os valores de $L^{*}$ pelo mecanismo supracitado, justificando a falta de diferença para esta variável.

A concentração e o estado da mioglobina são fatores preponderantes para os valores de $\mathrm{a}^{*}$. Os diferentes músculos são compostos por diferentes frequências dos tipos de fibras (JURIE et al., 2006), os quais possuem diferentes concentrações de mioglobina. Talvez, esta característica seja o principal fator responsável pelas diferenças verificadas para $a^{*}$ entre os cortes. O menor valor de $b^{*}$ para o Lombo não era esperado, pois este corte exibiu o maior teor de EE, e como mencionado antes, o valor $b^{*}$ está relacionado positivamente com o teor de gordura na carne.

$\mathrm{Na}$ avaliação da FC, observaram-se valores baixos para todos os cortes. $\mathrm{O}$ pernil obteve a maior força de cisalhamento, enquanto o lombo e a paleta não diferiram (Tabela 5). Dados de FC baixos em ovinos são normalmente encontrados, como no trabalho de Moreno et al. (2011), que apresentaram valores de FC variando de 0,99 a $1,22 \mathrm{kgf} / \mathrm{cm}^{2}$ no músculo Longissimus dorsi. FC reduzidos também foram encontrados por Pinheiro et al. (2009b), em diferentes músculos. Eles observaram que a paleta, independente da categoria animal apresentou-se como o corte menos macio (2 a $3,84 \mathrm{kgf} / \mathrm{cm}^{2}$ ). Múltiplos são os fatores que 
determinam com intensidade diferente a maciez nos músculos, como revisado por Thompson (2002). Nas condições deste estudo, independente da relação volumoso concentrado, o pernil foi $\mathrm{o}$ corte menos macio.

No geral, os músculos apresentaram claras diferenças. O lombo foi o corte com o menor valor para o parâmetro de cor $\mathrm{a}^{*}$, a paleta obteve o maior $\mathrm{pH}$ e o pernil apresentou o menor valor para o parâmetro $b^{*}$ e foi o corte menos macio. No entanto, apresentaram $\mathrm{pH}$ pouco acima da variação desejável $(5,4$ a 5,8), mas não houve comprometimento da qualidade da carne.

Quanto à composição centesimal, os cortes apresentaram pequenas diferenças para umidade, MM e PB. Entretanto, o EE apresentou diferença expressiva, onde o lombo apresentou o maior valor. Associando os dados obtidos, observa-se que o uso de uma ampla variabilidade de proporções de concentrado (de 20 a 80\%) pode ser utilizado na dieta, sem que grandes alterações sejam observadas na carne produzida.

O aumento dos níveis de concentrado não altera a composição centesimal das carnes e nem dos cortes cárneos, mas interfere nas características físicoquímicas, proporcionando carnes com menor luminosidade e mais vermelhas, mas que não comprometem o padrão de qualidade.

\section{REFERÊNCIAS}

ASSOCIATION OF OFFICIAL ANALYTICAL CHEMISTRY - AOAC. Official methods of analysis 16 . th. Arlington, 1995. p. 1025.
BICKERSTAFFE, R.; BEKHIT, A.E.D.; ROBERSTSON, L.J.; ROBERTS, N.; GEESINK, G.H. Impact of introducing specifications on the tenderness of retail meat. Meat science, v.59, p.303-315, 2001.

BONAGURIO, S.; PÉREZ, J.R.O.; FURUSHO-GARCIA, I.F.; BRESSAN, M.C.; LEMOS, A.L.S.C. Qualidade da carne de cordeiros Santa Inês puros e mestiços com Texel abatidos com diferentes pesos. Revista Brasileira de Zootecnia, v.32, n.6, p.1981-1991, 2003.

BONAGURIO, S.; PÉREZ, J.R.O.; FURUSHO-GARCIA, I.F.; SANTOS, C.L.; LIMA, A.L. Composição Centesimal da Carne de Cordeiros Santa Inês Puros e de seus Mestiços com Texel Abatidos com Diferentes Pesos. Revista Brasileira de Zootecnia, v.33, n.6, p.2387-2393, 2004.

CARVALHO, S.; MEDEIROS, L.M. Características de carcaça e composição da carne de cordeiros terminados em confinamento com dietas com diferentes níveis de energia. Revista Brasileira de Zootecnia, v.39, n.6, p.1295-1302, 2010.

COSTA, D.S.; COSTA, M.D.; SILVA, F.V.; ROCHA JÚNIOR, V.R.; CARVALHO, Z.G.; TOLENTINO, D.C.; LEITE, J.R.A. Desempenho ponderal de cordeiros Santa Inês e F1 Dorper x Santa Inês em pastagens naturais. Revista Brasileira de Saúde e Produção Animal [online], v.13, n.1, p.237-243, 2012.

COSTA, R.G.; LIMA, C.A.C.; MEDEIROS, A.N.; LIMA, G.F.C.L.; MARQUES, C.A.T.; QUEIROGA, R.C.R.E. Composição centesimal e análise sensorial da carne de ovinos Morada Nova alimentados com dietas contendo melão em substituição ao milho.

Revista Brasileira de Zootecnia, v.40, n.12, p.2799-2804, 2011a. 
COSTA, R.G.; SANTOS, N.M.; SOUSA, W.H.; QUEIROGA, R.C.R.E.; AZEVEDO, P.S.; CARTAXO, F.Q. Qualidade física e sensorial da carne de cordeiros de três genótipos alimentados com rações formuladas com duas relações volumoso:concentrado. Revista

brasileira de Zootecnia, v.40, n.8, p.1781-1787, 2011b.

DAVID, D.B.; POLI, C.H. E.C.; AZEVEDO, E.B.; FERNANDES, M.A.M.; CARVALHO, P.C.F.; JOCHIMS, F.; PIMENTEL, C.M.M.

Potential response to supplementation of ewe lambs grazing natural pastures over winter. Small Ruminant Research, v.105, p.22-28, 2012.

DEMIREL, G.; OZPINAR, H.; NAZLI, B.; KESER, O. Fatty acids of lamb meat from two breeds fed different forage: concentrate ratio. Meat Science, v.72, p.229-235, 2006.

EIKELENBOOM, G.; WALSTRA, P.; HUISKES, J.H. Species of meat animals/pigs. Encyclopedia of Meat

Sciences, v.3, p.1284-1291, 2004.

GONÇALVES, L.A.G.; ZAPATA, J.F.F.; RODRIGUES, M.C.P.; BORGES, A.S. Efeitos do sexo e do tempo de maturação sobre a qualidade da carne ovina. Ciência e Tecnologia de Alimentos, v.24, p.459-467, 2004.

'HONIKEL, K.O. Chemical and physical characteristics of meat: $\mathrm{pH}$ measument.

Encyclopedia of Meat Sciences, v.1, p.238-242, 2004.

JANSEN, M.L. Determination of meat $\mathrm{pH}$ : temperature relationship using ISFET and glass electrode instruments. Meat Science, v.58, p.145-150, 2001.

JURIE, C.; MARTIN, J-F; LISTRAT, A.; JAILLER, R.; CULIOLI, J.; PICARD, B. Carcass and muscle characteristics of beef cull cows between 4 and 9 years of age. Animal Science, v.82, n.3, p.415-421, 2006.

LAWRIE, R.A. Ciência da carne. 6.ed. Porto Alegre: Artmed, 2005. 384 p.

LEÃO, A.G.; SILVA SOBRINHO, A.G., MORENO, G.M.B.; SOUZA, H.B.A.; PEREZ, H.L.; LOUREIRO, C.M.B. Características nutricionais da carne de cordeiros terminados com dietas contendo cana-de-açúcar ou silagem de milho e dois níveis de concentrado. Revista Brasileira de Zootecnia, v.40, n.5, p.1072-1079, 2011.

MADRUGA, M.S.; SOUSA, W.H.; ROSALES, M.D.; CUNHA, M.G.G.; RAMOS, J.L.T. Qualidade da carne de cordeiros Santa Inês terminados com diferentes dietas. Revista Brasileira de Zootecnia, v.34, p.309-315, 2005.

MILLER, R. K. Chemical and physical characteristics of meat: palatability.

Encyclopedia of Meat Sciences, v.1, p.256-266, 2004.

MORENO, G.M.B.; BUZZULINI, C.; MORBA, H.; COSTA, A.J.; LIMA, T.M.A.; DOURADO, J.F.B. Efeito do genótipo e do teor de proteína da dieta sobre a qualidade da carne de cordeiros.

Revista Brasileira de Saúde e Produção Animal [online], v.12, n.3, p.630-640, 2011.

NATIONAL RESEARCH COUNCIL NRC. Nutrients requirements of small ruminants. Washington, D.C.: National Academy Press, 2007. 362p.

PFLANZER, S.B.; DE FELÍCIO, P.E. Moisture and fat content, marbling level and color of boneless rib cut from Nellore steers varying in maturity and fatness. Meat Science, v.87, n.1, p.7-11, 2011. 
PINHEIRO, R.S.B.; JORGE, A.M.; FRANCISCO, C.L.; ANDRADE, E.N. Composição química e rendimento da carne ovina in natura e assada. Ciência e Tecnologia de Alimentos, v.28, p.154$157,2008$.

PINHEIRO, R.S.B.; JORGE, A.M.; MOURÃO, R.C.; POLIZEL NETO, A.; ANDRADE, E.N.; GOMES, H.F.B. Qualidade da carne de cordeiros confinados recebendo diferentes relações de volumoso: concentrado na dieta.

Ciência e Tecnologia de Alimentos, v.29, n.2, p. 407-411, 2009a.

PINHEIRO, R.S.B.; SILVA SOBRINHO, A.G.; SOUSA, H.B.A.; YAMAMOTO, S.M. Qualidade de carnes provenientes de cortes da carcaça de cordeiros e de ovinos adultos. Revista Brasileira de Zootecnia, v.38, n.9, p.1790-1796, 2009b.

PRIOLO, A.; MICOL, D.; AGABRIEL, J.; PRACHE, S.; DRANSFIELD, E. Effect of grass or concentrate feeding systems on lamb carcass and meat quality. Meat science, v.62, n.2, p.179$185,2002$.

RIBEIRO, C.B. Exigência proteica de fêmeas ovinas mestiças. 2011. 77p. Dissertação (Mestrado em Ciência Animal) - Faculdade de Medicina Veterinária e Zootecnia, Universidade Federal de Mato Grosso do sul, Campo Grande.

SANTOS, J.R.S.D.; CEZAR, M.F.; SOUSA, W.H.D.; CUNHA, M.D.G.G.; PEREIRA FILHO, J.M.; SOUSA, D.O.D. Carcass characteristics and body components of Santa Inês lambs in feedlot fed on different levels of forage cactus meal. Revista Brasileira de Zootecnia, v.40, n.10, p.2273-2279, 2011.
SAÑUDO, C.; CAMPO, M.M.; SIERRA, I. Breed effect on carcase and meat quality of suckling lambs. Meat Science, v.46, n.4, p.357-365, 1997.

STATISTICAL ANALYSIS SYSTEM. Statistical analysis system: user's guide: statistics. Version 9.3. Cary: SAS Institute inc., 2011.

THOMPSON, J. Managing meat tenderness. Meat Science, v.62, n.3, p.295-308, 2002.

VIEIRA, T.R.L.; CUNHA, M.G.G.; GARRUTTI, D.S.; DUARTE, T.F.; FÉLEX, S.S.S.; PEREIRA FILHO, J.M.; MADRUGA, M.S. Propriedades físicas e sensoriais da carne de cordeiros Santa Inês terminados em dietas com diferentes níveis de caroço de algodão integral (Gossypium hirsutum). Ciência e Tecnologia de Alimentos, v.30, n.2, p.372-377, 2010.

WARNER, R.D.; GREENWOOD, P.L.; PETHICK, D.W.; FERGUSON, D.M. Genetic and environmental effects on meat quality. Meat Science, v.86, n.1, p.171-183, 2010.

WHEELER, T.L.; SHACKELFORD, S.D.; JOHNSON, L.P.; MILLER, M.F.; MILLER, R.K.; KOOHMARAIE, M. A comparison of Warner-Bratzler shear force assessment within and among institutions. Journal of Animal Science, v.75, p.2423-2432, 1997.

ZEOLA, N.M.B.L.; SILVA SOBRINHO, A.G.; GONZAGA NETO, S.G.

Composição centesimal da carne de cordeiros submetidos a dietas com diferentes teores de concentrado. Ciência Rural, v.34, n.1, p.253-257, 2004.

Data de recebimento: 11/06/2013

Data de aprovação: 05/12/2013 